\title{
MULTI-OBJECTIVE CONTROL OF A SELF-LOCKING COMPACT ELECTRO-HYDRAULIC CYLINDER DRIVE
}

\author{
Nikolaj Grønkær*, Lasse Nørby Nielsen, Frederik Ødum Nielsen, Søren Ketelsen and \\ Lasse Schmidt
}

Aalborg University, Department of Energy Technology, Pontoppidanstrcede 111, 9220 Aalborg East, Denmark

* Corresponding author: Tel.: +45 2966 7310; E-mail address: ngr@et.aau.dk

\begin{abstract}
The field of self-contained linear hydraulic drives based on variable-speed electrical motors and fixed displacement pumps is gaining interest from both industry and academia. Some of the main reasons for this is the possibility to improve the energy efficiency of such drives compared to conventional valve controlled drives, and the possibility for electrical regeneration allowing power sharing between multiple drives [1]. The main drawback for such types of drive concepts is a low pressure in the nonload carrying cylinder chamber. This induces a low drive stiffness limiting the achievable drive bandwidth and hence the application range. However, a so-called self-locking compact drive architecture recently proposed allows maintaining a proper drive stiffness by virtue of separate forward and return flow paths, combining the advantages of efficient flow control into the cylinder and a throttle driven flow out of the cylinder. The multiple inputs available in this architecture allow the control to target several objectives concurrently, for example piston motion, drive stiffness and fluid temperature. The purpose of the study presented is to analyse the dynamic couplings between the control objectives via relative gain array (RGA) methods, and the realization of input- and output transformations effectively decoupling relevant dynamic interactions. These transformations allow the usage of simple SISO-controllers for each control objective, and a method for controlling motion and fluid temperature concurrently, is proposed and experimentally verified.
\end{abstract}

Keywords: Input- and output transformations, self-contained hydraulic drives, speed-variable drives, multi-objective control, temperature control.

\section{INTRODUCTION}

The field of pump controlled electro-hydraulic differential cylinder drives is gaining increasing interest both in industry as well as in academia. Especially, actuation by variable-speed electrical drives combined with fixed displacement pumps has been a focus in the last decade [1]. The works on these electro-hydraulic drives have mainly been concerned with single pump closed circuit architectures or dual pump "open circuit" architectures [1-10]. Such electro-hydraulic drives have often been designed with on-board flexible reservoirs in terms of accumulators, making these drives hydraulically self-contained [1,6-10]. Furthermore, such drives feature a high degree of compactness compared to conventional valve drives supplied by hydraulic power units, and fast pump actuation dynamics dependent on the electrical motor and drive configuration. However, some main challenges with selfcontained electro-hydraulic drives are low drive stiffness due low pressure in the non-load carrying chamber and temperature control in the presence of a rather small fluid volume. As losses of such drives are generally low, both oil heating 


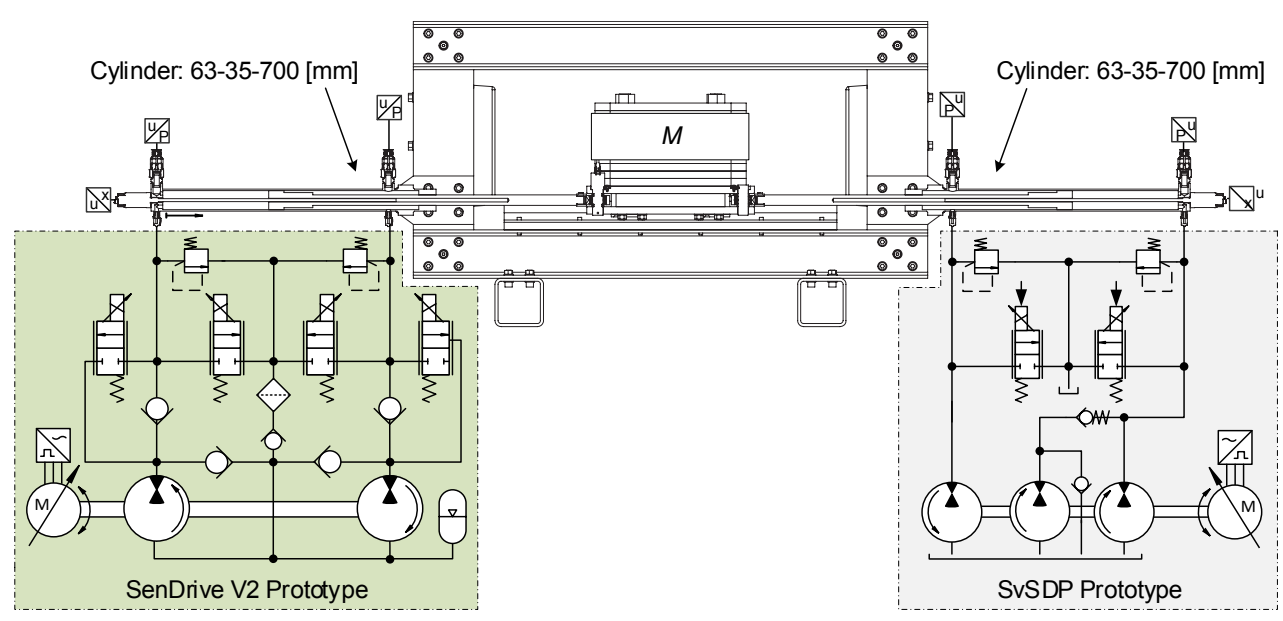

Figure 1: Test setup at Aalborg University, Denmark.

and cooling may be required to maintain the fluid temperature at some desired level.

The problem of low fluid stiffness has been addressed in several contributions, e.g. this was sought overcome by the so-called Speed-variable Switched Differential Pump (SvSDP) which is the three pump drive architecture [11-13], depicted in Fig. 1. Here the pump and cylinder volume flows are deliberately mismatched, causing the chamber pressures to increase when the pump leakage is overcome. The pressures may then be controlled to an acceptable level by means of throttling of the excess flow, hence drive stiffness is achieved on the cost of energy efficiency. A main drawback of this drive (and other pump controlled drives) is the necessity to compensate pump leakage when the cylinder piston is stationary since this induces power consumption of the electric motor.

An initiative to limit this problem was introduced with a "self-locking" electrohydraulic drive $[14,15]$, the so-called SenDrive also depicted in Fig. 1. This drive separates the flow paths into and out of the cylinder, such that pump flow is passed to the cylinder via rectifying valves and extracted from the cylinder via proportional throttle valves. The drive features a common return flow path allowing for filtering and potentially also cooling of the return flow fluid, or the return flows may bypass the rectifying valves allowing to regenerate energy in load aiding quadrants. Furthermore, as for the SvSDP drive, the pressure level, hence the drive stiffness, may be controlled to a desired level on the cost of energy efficiency. This is the case since the throttling is used to maintain a certain pressure level. However, this induces losses, which elevates the temperature with a certain rate dependent on the pressure level and the velocity/flow, which induces a coupling between the temperature, the drive stiffness and motion.

The five inputs of the drive allow reaching multiple control objectives concurrently, e.g. control of the pressure level and piston position or force. However, as the throttle losses are controllable, the temperature may also be increased to a desired level when heating is needed, while controlling motion or force.

This paper is concerned with a model based control design for the SenDrive architecture discussed above, encompassing decoupling of the main dynamic couplings between the cylinder pressure level and the piston motion, combined with control loops allowing to control the piston position as well as heating of the fluid concurrently. The latter is realized by controlling the pressure level and hence the throttling losses, where the pressure level reference is realized by a temperature control loop, effectively realizing a cascade control structure. The control design is experimentally verified, and results demonstrate that accurate control of the motion and fluid heating is achieved. 


\subsection{Application for study}

All the experimental tests have been conducted on the test bench depicted in Fig. 1, and the main components of the SenDrive prototype are listed in Tab. 1, which is located at Aalborg University, Aalborg Denmark. The main focus of the paper is the SenDrive prototype, whereas the SvSDP prototype is operating as a load. In the model this load is seen as a constant force as seen in Fig. 2. The model parameters used in the following, are verified with experimental data and given in the nomenclature shown in Tab. 2.

Table 1: Main components of SenDrive prototype drive. ${ }^{a}$ Bosch Rexroth AG, ${ }^{b}$ Fjero $\mathrm{A} / \mathrm{S}$, 'Balluf GmbH

\begin{tabular}{ll}
\hline Component & Description \\
\hline${ }^{\mathrm{a}}$ MOT-FC-ET2 & Induction motor \\
${ }^{\mathrm{a}}$ IndraDrive HCS03-70A & Converter \\
${ }^{\mathrm{a}}$ CSH02.1B & Control unit \\
${ }^{\mathrm{a}}$ AZPFFF-12-016/011 & Pump rack \\
${ }^{\mathrm{a}}$ KKDSR1NB & $2 / 2$ prop. Valves \\
${ }^{\mathrm{a}}$ HM 20-2X/250 & Pres. sensor(s) \\
${ }^{\mathrm{b}}$ Cylinder 63-35-700 & Hyd. cylinder \\
${ }^{\mathrm{c} B T L 7 ~-~ S ~ 5 ~ 0 ~}$ & Position sensor \\
\hline
\end{tabular}

\section{MODEL}

The model of the SenDrive prototype used in the study is constituted by a hydraulic/mechanical model, which is extended with a thermal model representing the average temperature dynamics.

\subsection{Hydraulic model}

The hydraulic model of the SenDrive prototype takes offset in the schematic depicted in Fig. 2, and is given by Eqs. (1)-(13), where $j=v A, v B$, $v A T, v B T$ and $i=A T, B T, T A, T B, R$ and $k=A, B$, $A T, B T, R, T$ and $\Delta \mathrm{x}_{v A}=\mathrm{x}_{v A}-\mathrm{x}_{d}, \Delta \mathrm{x}_{v B}=\mathrm{x}_{v B}-\mathrm{x}_{d}$, $\Delta \mathrm{x}_{v A T}=\mathrm{x}_{v A T}-\mathrm{x}_{d}, \Delta \mathrm{x}_{v B T}=\mathrm{x}_{v B T}-\mathrm{x}_{d}$.

$$
\begin{aligned}
& \ddot{x}_{p}=\frac{1}{M}\left(A_{A} P_{L}-B_{v} \dot{x}_{p}-F_{e x t}\right), P_{L}=P_{A}-\alpha P_{B} \\
& \dot{P}_{A}=\frac{\beta_{A}}{V_{A}}\left(Q_{A T}-Q_{v A T}-Q_{v A}-\dot{x}_{p} A_{A}\right) \\
& \dot{P}_{B}=\frac{\beta_{B}}{V_{B}}\left(Q_{B T}-Q_{v B T}-Q_{v B}+\dot{x}_{p} A_{B}\right) \\
& \dot{P}_{A T}=\frac{\beta_{A T}}{V_{A T}}\left(Q_{T A}+Q_{p A}+Q_{v A T}-Q_{A T}\right) \\
& \dot{P}_{B T}=\frac{\beta_{B T}}{V_{B T}}\left(Q_{T B}-Q_{p B}+Q_{v B T}-Q_{B T}\right)
\end{aligned}
$$

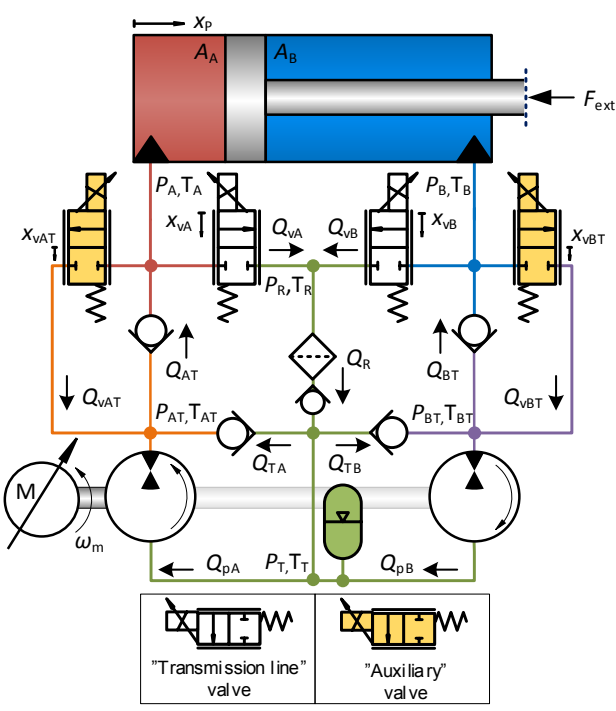

Figure 2: Schematic of Self-locking compact electrohydraulic cylinder drive with model nomenclature.

$\dot{P}_{R}=\frac{\beta_{R}}{V_{R}}\left(Q_{v A}+Q_{v B}-Q_{R}\right)$

$\dot{P}_{T}=\frac{\beta_{T}}{V_{T}+\frac{\beta_{T} V g}{\kappa P_{T}}}\left(Q_{R}-Q_{p A}+Q_{p B}-Q_{T A}-Q_{T B}\right)$

$\ddot{x}_{j}=\omega_{v}^{2}\left(u_{j}-x_{j}\right)-2 \zeta_{v} \omega_{v} \dot{x}_{j}$

$\ddot{\omega}_{m}=\omega_{e}^{2}\left(u_{m}-\omega_{m}\right)-2 \zeta_{e} \omega_{e} \dot{\omega}_{m}$

$Q_{p A}=D_{A} \omega_{m}-C_{L A}\left(P_{A T}-P_{T}\right)$

$Q_{p B}=D_{B} \omega_{m}+C_{L B}\left(P_{B T}-P_{T}\right)$

$Q_{v A}=\left\{\begin{array}{cc}\Delta x_{v A} k_{v A} \tanh \left(\left(P_{A}-P_{R}\right) k_{p}\right) & , \Delta x_{v A} \geq 0 \\ 0 & , \Delta x_{v A}<0\end{array}\right.$

$Q_{v B}=\left\{\begin{array}{cc}\Delta x_{v B} k_{v B} \tanh \left(\left(P_{B}-P_{R}\right) k_{p}\right) & , \Delta x_{v B} \geq 0 \\ 0 & , \Delta x_{v B}<0\end{array}\right.$

$Q_{v A T}=\left\{\begin{array}{cl}\Delta x_{v A T} k_{v A T} \tanh \left(\left(P_{A}-P_{A T}\right) k_{p}\right) & , \Delta x_{v A T} \geq 0 \\ 0 & , \Delta x_{v A T}<0\end{array}(14)\right.$

$Q_{v B T}=\left\{\begin{array}{cl}\Delta x_{v B T} k_{v B T} \tanh \left(\left(P_{B}-P_{B T}\right) k_{p}\right) & , \Delta x_{v B T} \geq 0 \\ 0 & , \Delta x_{v B T}<0\end{array}\right.$

$V_{A}=V_{A 0}+x_{p} A_{A}, V_{B}=V_{B 0}+\left(L_{x}-x_{p}\right) A_{B}$

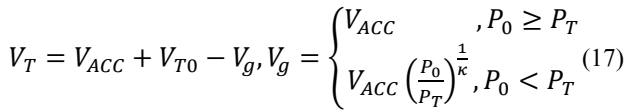

$Q_{A T}=\left\{\begin{array}{cl}K_{A T} x_{A T} \sqrt{P_{A T}-P_{A}} & , P_{A T} \geq P_{A} \\ 0 & , P_{A T}<P_{A}\end{array}\right.$

$Q_{B T}=\left\{\begin{array}{cl}K_{B T} x_{B T} \sqrt{P_{B T}-P_{B}} & , P_{B T} \geq P_{B} \\ 0 & , P_{B T}<P_{B}\end{array}\right.$

$Q_{T A}=\left\{\begin{array}{cc}K_{T A} x_{T A} \sqrt{P_{T}-P_{A T}} & , P_{T} \geq P_{A T} \\ 0 & , P_{T}<P_{A T}\end{array}\right.$ 


$$
\begin{aligned}
& Q_{T B}=\left\{\begin{array}{cc}
K_{T B} x_{T B} \sqrt{P_{T}-P_{B T}} & , P_{T} \geq P_{B T} \\
0 & , P_{T}<P_{B T}
\end{array}\right. \\
& Q_{R}=\left\{\begin{array}{cc}
K_{R} x_{R} \sqrt{P_{R}-P_{T}} & , P_{R} \geq P_{T} \\
0 & , P_{R}<P_{T}
\end{array}\right. \\
& x_{i}=\left\{\begin{array}{ccc}
0 & , & \Delta P_{c v}<P_{c v, c r} \\
\frac{\Delta P_{c v}-P_{c v, c r}}{P_{c v, \text { end }}-P_{c v, c r}} & , P_{c v, c r} \leq \Delta P_{c v}<P_{c v, \text { end }} \\
1 & , & P_{c v, \text { end }} \leq \Delta P_{c v}
\end{array}\right. \\
& \beta_{k}=\frac{1}{\frac{1}{\beta_{F}}+}\left(\frac{1}{\left.\left(1-\eta_{\text {air }}\right) \frac{\left(\frac{P_{\text {atm }}}{P_{k}+P_{\text {atm }}}\right)^{-\frac{1}{\kappa}}}{\eta_{\text {air }}}+1\right) \kappa\left(P_{k}+P_{\text {atm }}\right)}\right.
\end{aligned}
$$

\subsection{Thermal model extension}

The thermal model extension is constructed as an average model of the temperature dynamics, as the drive level temperature time constant may be considered strictly larger than the remaining time constants of the drive. The internal temperature variations are uncontrollable due to the internal restrictions and flow paths, but will fluctuate about the average temperature. Assuming a constant ambient temperature, the average temperature dynamics is governed by the hydraulic losses, natural convection, forced

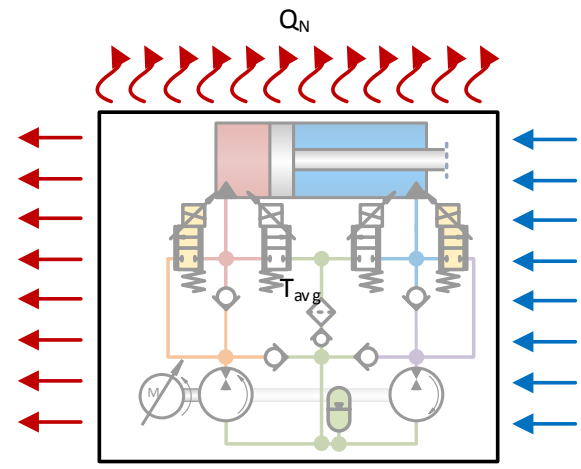

(A)

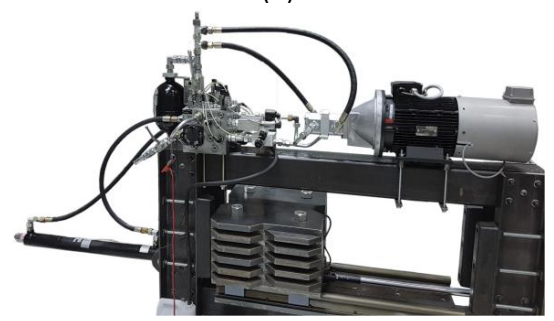

(B)

Figure 3: Thermal model principal for the thermal extension and photo of test setup. convection and radiation. Forced convection is induced by the electric motor cooling fan, also blowing air across the manifold and pumps besides the motor (see Fig. 3. (B)). Hence, an average temperature model for drive may be established as Eqs. (25), (26).

$$
\begin{aligned}
& \dot{T}_{\text {avg }}=\frac{\dot{W}_{\text {loss }}-h_{t}\left(T_{\text {avg }}-T_{\infty}\right)}{c_{e q} m_{e q}}, h_{t}=h_{n}+h_{f} \\
& \dot{W}_{\text {loss }}=P_{\text {in }}-P_{\text {out }}=\omega_{m} \tau_{t}-P_{L} A_{A} \dot{x}_{p}
\end{aligned}
$$

Note that the thermal model is highly simplified compared to a full model of the thermal behaviour of such a drive (see e.g. [7]). However, in terms of usage of the model for temperature level control design, and the large time constant of the temperature dynamics, the model is considered sufficiently accurate for the purpose of this study.

\section{COUPLING ANALYSIS}

In the coupling analysis presented in the following, only the case of piston extension is considered for brevity. Furthermore, all check valves are assumed ideal, which is reasonable since the pressure drops across the valves at maximum flow are less than 1 [bar].

Considering Fig. 2. under the above mentioned assumption and piston extension, then $\dot{P}_{A T}=$ $\dot{P}_{A} \rightarrow \beta_{A}=\beta_{A T}$. Combining Eqs. (2), (4) under these considerations, Eq. (27) is obtained.

$\dot{P}_{A}=\frac{\beta_{A}}{V_{A}+V_{A T}}\left(Q_{P A}-Q_{v A}-\dot{x}_{p} A_{A}\right)$

Furthermore, the following study is restricted to the usage of the "transmission line" valves, hence, under the assumption of ideal check valves, Eq. (28) is valid.

$P_{B T}=P_{T}, P_{R}=P_{T}, Q_{v B T}=Q_{v A T}=0$

Finally, to avoid unnecessary power loss, then under piston extension $Q_{v A}=0$ is maintained. With these considerations, the drive dynamics under piston extension may be simplified to Eqs. (29)-(32).

$$
\begin{aligned}
& \ddot{x}_{p}=\frac{1}{M}\left(A_{A} P_{L}-B_{v} \dot{x}_{p}\right), P_{L}=P_{A}-\alpha P_{B} \\
& \dot{P}_{A}=\frac{\beta_{A}}{V_{A}+V_{A T}}\left(Q_{p A}-\dot{x}_{p} A_{A}\right) \\
& \dot{P}_{B}=\frac{\beta_{B}}{V_{B}}\left(-Q_{v B}+\dot{x}_{p} A_{B}\right)
\end{aligned}
$$


$\dot{T}_{\text {avg }}=\frac{\dot{W}_{\text {loss }}-h_{t}\left(T_{\text {avg }}-T_{\infty}\right)}{c_{e q} m_{e q}}$

Linearizing the model Eqs. (29)-(32) and Eqs. (9)-(11), a combined linear model for the case of piston extension may be established similar to [11], as the transfer function matrix Eq. (33). Compared to [11] this model extended with the temperature and the ratio between the cylinder chamber bulk modulii.

$\left[\begin{array}{c}\dot{x}_{p}(s) \\ p_{A}(s) \\ p_{B}(s) \\ T_{\text {avg }}(s)\end{array}\right]=\boldsymbol{G}(s)\left[\begin{array}{l}u_{m}(s) \\ u_{v B}(s)\end{array}\right]$
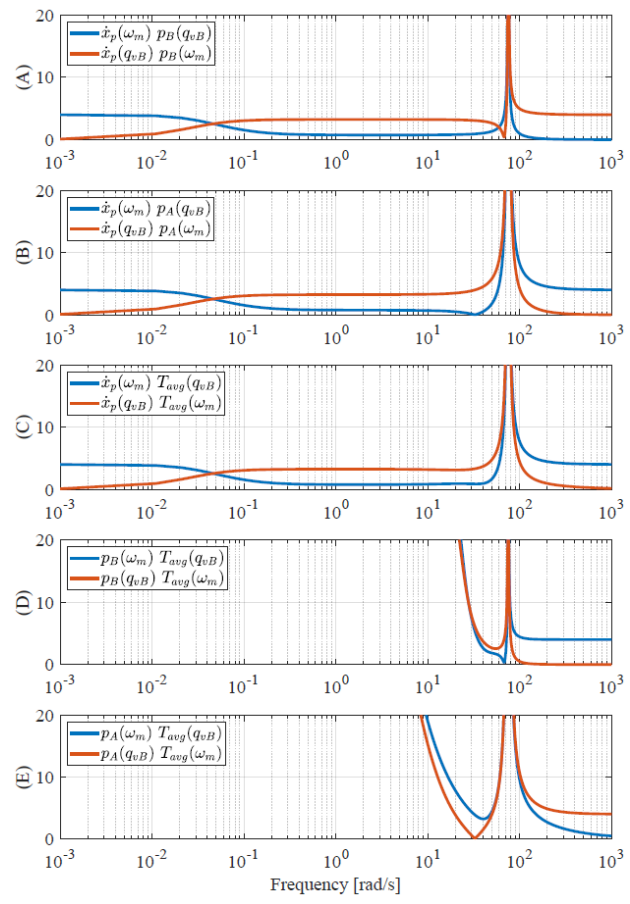

Figure. 4: Relative gain array for system with actuator dynamics.

Using linearization points $x_{p 0}=350[\mathrm{~mm}]$, $P_{A 0}=35[\mathrm{bar}], \quad P_{B 0}=5[\mathrm{bar}], \dot{x}_{p 0}=$ $125[\mathrm{~mm} / \mathrm{s}], \omega_{m 0}=100[\mathrm{rad} / \mathrm{s}]$, i.e. $F=$ $10.7[k N]$, the RGA numbers depicted in Fig. 4 may be established. Bearing in mind that in the event of ideally decoupled dynamics, the RGA numbers will be 0 and 4 , it is evident that heavy couplings are present, as may be expected. These couplings may complicate control design significantly when targeting the control of both the motion and pressure level concurrently.
Hence, in the following a decoupling strategy is proposed, allowing the usage of decentralized control. RGA numbers for different piston positions are depicted in the Appendix.

\section{DECOUPLING}

The decoupling approach of the drive follows the idea presented in [11]. The utilised control structure is seen in Fig. 5, where the input and output transformations are based on the load pressure $P_{L}$ and a "level pressure" $P_{H}$ used as the outputs with the corresponding load and level "flows", $Q_{L}$ and $Q_{H}$, used as inputs.

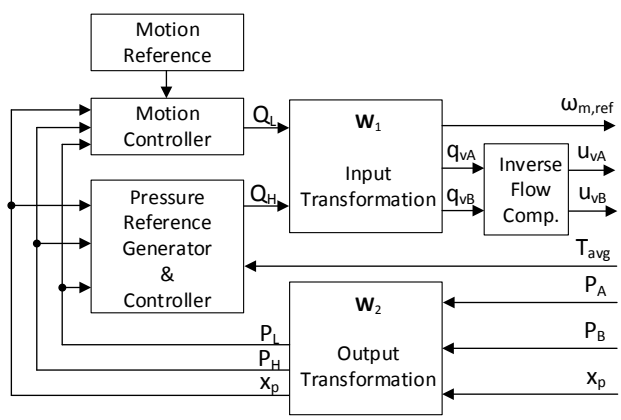

Figure 5: General control structure.

\subsection{Output Transformation}

The load and level pressures are defined as Eq. (34) with the corresponding dynamics given by Eq. (35).

$P_{L}=P_{A}-\alpha P_{B}, \quad P_{H}=P_{A}+H P_{B}$

$\dot{P}_{L}=\dot{P}_{A}-\alpha \dot{P}_{B}, \quad \dot{P}_{H}=\dot{P}_{A}+H \dot{P}_{B}+\dot{H} P_{B}$

With the definitions in Eq. (34), the cylinder chamber pressures may be expressed as Eq. (36). $P_{A}=\frac{H P_{L}+\alpha P_{H}}{H+\alpha}, \quad P_{B}=\frac{P_{H}-P_{L}}{H+\alpha}$

In [11] it is assumed that $\beta_{A}=\beta_{B}$, which is sensible if $P_{A}, P_{B}>20$ bar. However, the temperature control and efficiency depend on a possibility to control the non-load carrying pressure to lower levels, hence one may assert relation Eq. (37), where $\epsilon$ is the pressure dependent bulk modulus ratio.

$\beta_{B}=\epsilon\left(P_{A}, P_{B}\right) \beta_{A}$ 
Using this ratio, the pressure dynamics Eq. (35) may be expressed with Eqs. (30), (31) as Eqs. (38), (39), noting Eq. (40).

$$
\begin{aligned}
\dot{P}_{L} & =\frac{\beta_{A}\left(\rho V_{A}\left(Q_{p A}-A_{A} \dot{x}_{p}\right)-\left(A_{A} \alpha \dot{x}_{p}-Q_{v B}\right) V_{A P} \alpha \epsilon\right)}{V_{A P} \rho V_{A}} \\
\dot{P}_{H} & =\frac{\beta_{A}\left(A_{A}\left(H \alpha \epsilon V_{A P}-\rho V_{A}\right) \dot{x}_{p}-Q_{v B} V_{A P} H \epsilon-\rho V_{A} Q_{P A}\right)}{\rho V_{A} V_{A P}} \\
+ & \frac{\left(P_{H}-P_{L}\right) \dot{H}}{H+\alpha} \\
V_{A P} & =V_{A}+V_{A T}, \rho=\frac{V_{B}}{V_{A}}
\end{aligned}
$$

From the level pressure dynamics Eq. (39), it is notable that $H$ influences the volume flow. Hereby, it is found sensible to choose $H=\frac{\rho V_{A}}{\mu \alpha V_{A P}}$ where $\mu$ approximates $\epsilon$. This approximation is made by a simplified version of the bulk modulus ratio model (Eqs. (24), (37)) and is given by Eq. (41).

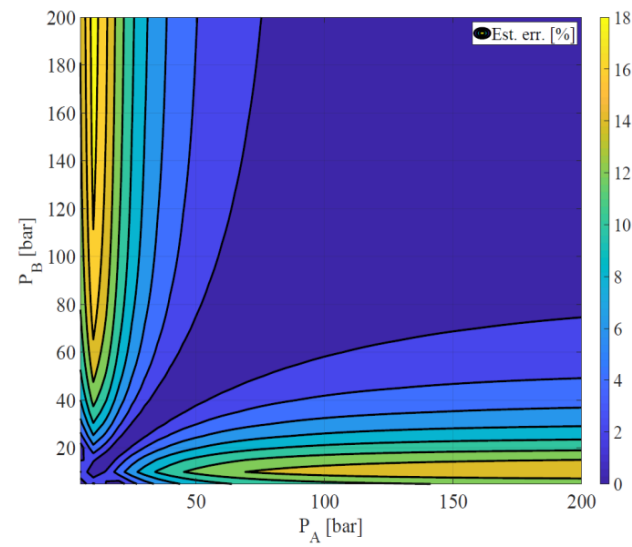

Figure 6: Relative estimation error of the bulk modulus ratio $(\mu)$.

$\mu=\frac{\left.\left(P_{B}+P_{a t m}\right)^{2}\left(\left(\eta_{a i r}+1\right) P_{a t m}^{2}+\left(\left(P_{A}+\beta_{F}\right) \eta_{a i r}+2 P_{A}\right) P_{a t m}+P_{A}^{2}\right)\right)}{\left.\left(P_{A}+P_{a t m}\right)^{2}\left(\left(\eta_{a i r}+1\right) P_{a t m}^{2}+\left(\left(P_{B}+\beta_{F}\right) \eta_{a i r}+2 P_{A}\right) P_{a t m}+P_{B}^{2}\right)\right)}$

With this estimation a maximal estimation error for $\mu$ is $20 \%$ in the operational range for the system $P_{A}, P_{B} \leq 200$ [bar], as depicted in Fig. 6 . Furthermore, Fig. 6 shows that the estimation error increases if one of the pressures is rather low and a large difference between the pressures is present. From this it is found that the estimation error has the largest magnitude when the load pressure is high and when the non-load carrying pressure is low.

Since the air percentage in the oil is a sensitive parameter, the sensitivity of air in the fluid is investigated. The same map as in Fig. 6 is depicted in Fig. 7 with the air percentage in the oil doubled $\left(\eta_{\text {air }}=2 \eta_{\text {air }}\right)$. It is seen that the maximum error increases to $25 \%$, which indicates that the estimation is fairly insensitive to a parameter error on the air content in the oil. Furthermore, it is seen that the error is largest when the load pressure is negative.

If an ideal approximation of the bulk modulus ratio $(\mu=\epsilon)$ is assumed and it is assumed that the ratio is slow varying $(\dot{\epsilon} \approx 0)$, the level and load pressure dynamics are simplified to Eqs. (42), (43), (44), with $V_{A \max }=A_{A} L+V_{A 0}$.

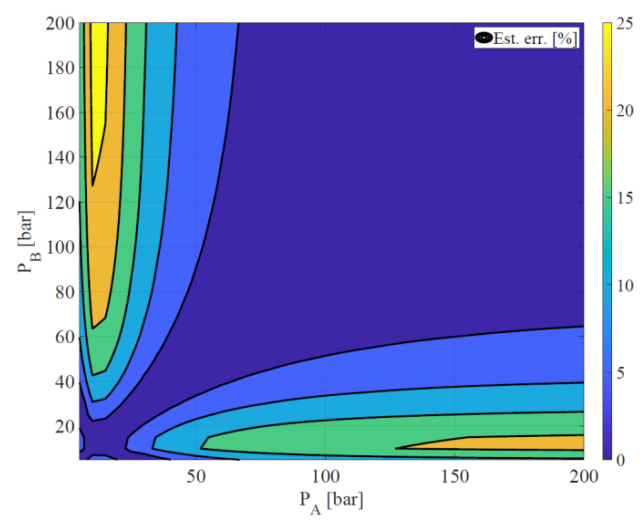

Figure 7: Relative estimation error of the bulk modulus ratio $(\mu)$ with twice the amount of air $\left(\eta_{\text {air }}=2 \eta_{\text {air }}\right)$. 


$$
\begin{aligned}
& \dot{P}_{L}=\frac{\beta_{A}\left(\rho V_{A}\left(Q_{p A}-A_{A} \dot{x}_{p}\right)-\left(A_{A} \alpha \dot{x}_{p}-Q_{v B}\right) V_{A P} \alpha \epsilon\right)}{V_{A P} \rho V_{A}} \\
& \dot{P}_{H}=\frac{\beta_{A}\left(\alpha Q_{P A}-Q_{v B}\right)}{\alpha V_{A P}}+\frac{\left(P_{H}-P_{L}\right) \dot{H}}{H+\alpha} \\
& \dot{H}=-\frac{\left(\left(V_{A \max }+V_{A T}\right) \alpha+V_{B 0}\right)}{V_{A P}^{2} \mu \alpha} \dot{x}_{p}
\end{aligned}
$$

\subsection{Input Transformation}

A steady state decoupling may be established, considering the inputs $\omega_{m}=\frac{A_{A}}{P} \bar{\omega}_{m}$ and $Q_{v B}$, and defining a "load flow" Eq. $45^{A}$ and "level flow" Eq. (46), constructed from the actuation flows, then the load and level pressures may be described as Eqs. (47),(48), such that the coupled input effects are "hidden" from the pressure dynamics.

$Q_{L}=\frac{\alpha \epsilon V_{A P}\left(A_{A} \alpha \bar{\omega}_{m}+Q_{v B}\right)+A_{A} V_{A} \rho \bar{\omega}_{m}}{\alpha^{2} \epsilon V_{A P}+\rho V_{A}}$

$Q_{H}=-Q_{v B}$

$\dot{P}_{L}=\frac{\beta_{A}\left(\alpha^{2} \epsilon V_{A P}+\rho V_{A}\right)\left(Q_{L}-A_{A} \dot{x}_{p}\right)}{V_{A P} \rho V_{A}}-$

$\frac{\beta_{A} C_{L A}\left(\alpha^{2} \epsilon V_{A P} P_{H}+P_{L} \rho V_{A}\right)}{V_{A P}\left(\alpha^{2} \epsilon V_{A P}+\rho V_{A}\right)}$

$\dot{P}_{H}=\frac{\beta_{A} Q_{H}}{V_{A P} \alpha}-\frac{\beta_{A} C_{L A}\left(\alpha^{2} \epsilon P_{H} V_{A P}+P_{L} V_{A} \rho\right)}{\left(\alpha^{2} \epsilon V_{A P}+\rho V_{A}\right) V_{A P}}+\frac{\left(P_{H}-P_{L}\right) \dot{H}}{H+\alpha}$

The control designs may now be conducted in the load and level pressure domain, i.e. for $Q_{L}$ and $Q_{H}$, and then transformed to the physical inputs by Eq. (49).

$Q_{v B}=-Q_{H} \quad \bar{\omega}_{m}=\frac{Q_{L}}{A_{A}}+\frac{Q_{H}}{A_{A}(H+\alpha)}$

\subsection{Decoupling analysis}

In order to verify the impact of the decoupling approach, an additional RGA analysis is carried out for the transformed system with the actuator dynamics, i.e. the system described by Eqs. (1), (9), (10), (47), (48). Similar to Section 3, this system is linearized (at the same linearization point), and a transfer function matrix $\widetilde{\boldsymbol{G}}(s)$ may be established, satisfying Eq. (50), where $p_{L}, p_{H}, q_{L}, q_{H}$ denote the change variables of $P_{L}, P_{H}, Q_{L}, Q_{H}$.

$$
\left[\begin{array}{c}
\dot{x}_{p}(s) \\
p_{L}(s) \\
p_{H}(s) \\
T_{\text {avg }}(s)
\end{array}\right]=\widetilde{\boldsymbol{G}}(s)\left[\begin{array}{c}
q_{L}(s) \\
q_{H}(s)
\end{array}\right]
$$

The results of the analysis are depicted in Fig. 8, noting that $\mu \neq \epsilon$ and Eq. (41) is used in the transformations. From this it is found that the motion and level pressure are nearly completely decoupled throughout the entire frequency range. However, it seen that coupled effects still are present between the level pressure, motion and the temperature. Especially the level pressure and temperature have a large coupling during the lower frequency range. In a physical sense, this is also expected since the throttle losses increase with the level pressure and hereby the amount of energy dissipated to the oil/system. Furthermore, it is seen that the motion/flow also couples with the temperature in the higher frequency range, which is sensible since the power loss produced by throttling depends on both pressure drop and flow/velocity and hereby motion. Further working points with a varying piston position are depicted in the Appendix for the decoupled system.

From these results, it is found that decentralized (SISO) control designs may be applied for motion and level pressure control, and
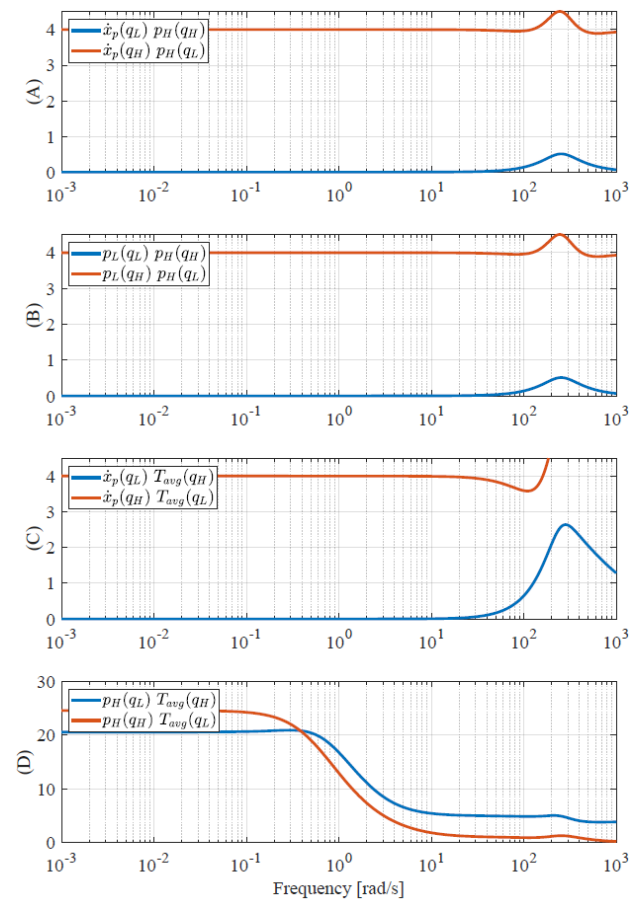

Figure 8. Relative gain array for system with inputand output transformations. 
that the level pressure reference may be established as a function of the desired temperature, since the level pressure is highly linked to the losses. In the application of this approach, the pressure level will be dictated by the desired temperature and not e.g. desired stiffness, which also would be possible [14].

\section{CONTROL DESIGN}

Besides the decoupling methodology, simple proportional control is used for the motion, level pressure and temperature controllers in the decoupled domain. The level pressure and temperature controllers are constructed as a cascade structure, as the output of the temperature controller is the reference to the level pressure controller. The motion controller is a combination of proportional control, velocity feed forward and active damping.

\subsection{Level pressure control}

The idea behind the level pressure control was originally to control the drive stiffness by controlling the non-load carrying chamber pressure to a certain pressure setting, i.e. such that $\min \left\{P_{A}, P_{B}\right\}=P_{\text {set }}$. From this definition, a switch condition is defined as Eq. (51).

$P_{A}=P_{B}=P_{\text {set }} \rightarrow P_{L, s w}=P_{\text {set }}(1-\alpha)$

$P_{L} \geq P_{L, s w} \rightarrow P_{A}=P_{L}+\alpha P_{\text {set }}, P_{B}=P_{\text {set }}$

$P_{L}<P_{L, s w} \rightarrow P_{A}=P_{\text {set }}, P_{B}=\frac{P_{\text {set }}-P_{L}}{\alpha}$

The level pressure reference is then generated from the definition of the level pressure Eq. (34), given by Eq. (52).

$P_{H, r e f}= \begin{cases}P_{L}+(H+\alpha) P_{\text {set }} & P_{L} \geq P_{L, s w} \\ -\frac{H}{\alpha} P_{L}+\frac{\alpha+H}{\alpha} P_{\text {set }} & P_{L}<P_{L, s w}\end{cases}$

From this, the level pressure control error is defined as Eq. (53).

$e_{H}= \begin{cases}(H+\alpha)\left(P_{\text {set }}-P_{B}\right) & P_{L} \geq P_{L, s w} \\ \frac{\alpha+H}{\alpha}\left(P_{\text {set }}-P_{A}\right) & P_{L}<P_{L, s w}\end{cases}$

The proportional level pressure controller is given by Eq. (54).

$Q_{H}=k_{p p} e_{H}$
Note that the switch of the reference Eq. (53) is bump-less, since $e_{H}=0$ when the switch occurs.

\subsection{Motion Control}

The position control is realized via the load flow. The position controller is constituted by a proportional position control term, a velocity feed forward and an active damping term since the drive is under damped. The latter is realized by Eqs. (55), (56).

$Q_{L}=\dot{x}_{p, r e f} A_{A}+k_{p x} e_{x}-k_{a d} P_{L}^{*}$

$P_{L}^{*}=P_{L} \frac{s}{\left(s+\omega_{c}\right)\left(s+\omega_{f}\right)}$

In Eq. (56) the filter frequencies are chosen such that $\omega_{c}<$ drive eigenfrequency $<\omega_{f}<$ measurement noise frequency. This allow pressure transients to pass the filter while attenuating the load pressure level and noise.

\subsection{Temperature control}

The idea behind the temperature control is that the losses can be controlled directly via the level pressure setting $P_{\text {set }}$, and through this control the temperature, which is justified by Fig 8 (D). From the RGA in Fig 7 (C) it is found that the temperature and motion dynamics are decoupled, indicating that temperature control is possible without compromising the motion control. Note that the temperature controlled is the average temperature hence variation in the temperature may be present internally in the drive. However, it is assumed that these variations are of less importance. The proportional controller Eq. (57) is used to generate the pressure set point.

$P_{\text {set }}^{*}=\left(T_{\text {set }}-T_{\text {avg }}\right) k_{p t}$

To ensure that the pressure is maintained within a reasonable range, a saturation is made on the pressure set point as Eq. (58).

$P_{\text {set }}=\left\{\begin{array}{lr}P_{\text {set,max }}, & P_{\text {set }}^{*} \geq P_{\text {set, } \text { max }} \\ P_{\text {set }}^{*}, & \mathrm{P}_{\text {set,min }} \leq \mathrm{P}_{\text {set }}^{*} \leq P_{\text {set,max }} \\ P_{\text {set,min },} & P_{\text {set }}^{*} \leq P_{\text {set }, \text { min }}\end{array}\right.$ 
Note that Eq. (58) limits the temperature range, especially if the ambient temperature is high such that the natural cooling is low. Hence, the system is forced to be more efficient (lower $P_{s e t}$ ) to maintain the temperature low.
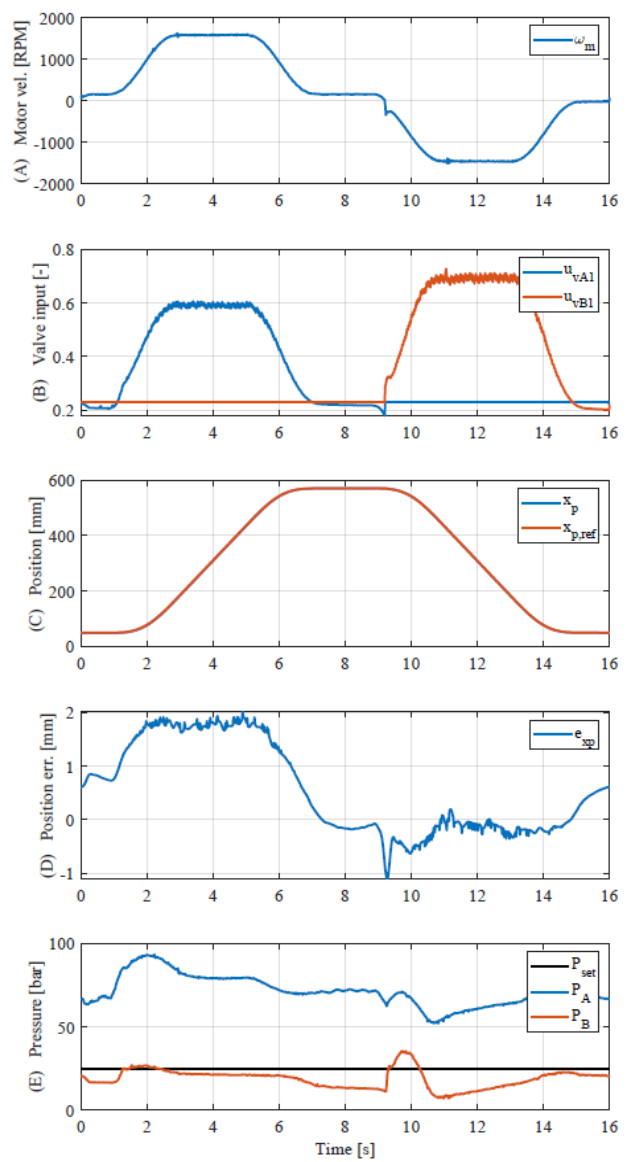

Figure 9: Experimental results from test of position and pressure controller with an external force $F_{\text {ext }}=20 \mathrm{kN}$ and a maximal piston velocity of $125 \mathrm{~mm} / \mathrm{s}$.

\section{EXPERIMENTAL VERIFICATION}

The experimental results are shown in Fig. 9, for a test with an external force of $20[\mathrm{kN}]$ and a maximal piston velocity of $125[\mathrm{~mm} / \mathrm{s}]$. It is seen that the simple controllers maintain a rather accurate position control with a maximal position error of 2 [mm]. Furthermore, a steady state error is present, which also indicates that a proportional controller may be insufficient for high performance control demands. However, the decoupling allows the usage of two simple decentralised controllers as intended.

In Fig. 10 the results of the temperature control are shown. This test is a repeating sequence of the test used in Fig 9. A steady state error is expected due to the choice of controller, which also is seen in the figure. However, it is seen in Fig. 10 (A) that all the temperatures of the drive lies with a maximum deviation of $\pm 2{ }^{\circ} \mathrm{C}$, which is an acceptable variation of the temperature throughout the system (Bosch Rexroth Denmark). Furthermore, it is seen in Fig. 10 (B) that the pressure is lowered as intended when the temperature is rising. Note that this temperature control has the limitations:

- Upper pressure limit restricts the maximal loss and hereby sets an upper bound on the temperature depending on free and forced convection.

- Lower pressure limit restricts the minimum loss and hereby sets a lower bound on the temperature depending on free and forced convection.

- A high drive stiffness requirement restricts the lower pressure bound even further and hereby the lower bound for the temperature.

- The force and piston velocity limits the temperature range since the throttling depends on these and hereby the amount of power dissipated.

From the latter, it is concluded that the temperature control works as intended, but if the compromise between the temperature control and the drive stiffness should be eliminated, an 
additional control input is needed in form of an active cooler
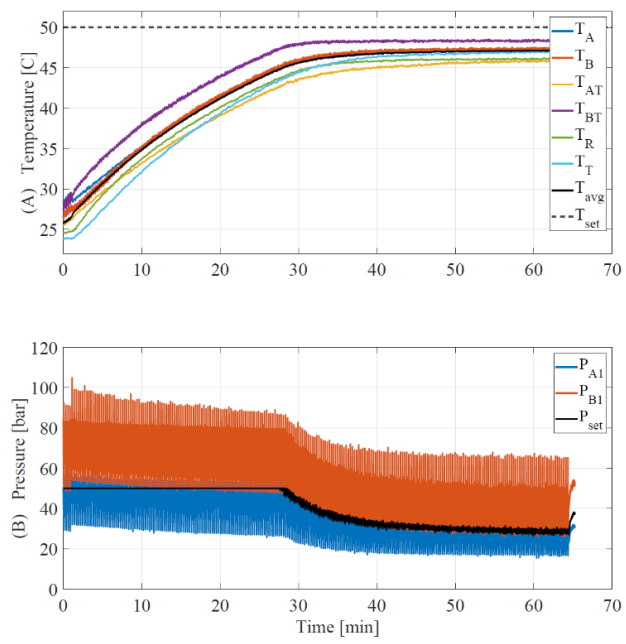

Figure 10. Test results from test of temperature control with an external force of $F_{\text {ext }}=0 \mathrm{kN}$ and a maximal piston velocity of $125 \mathrm{~mm} / \mathrm{s}$.

\section{CONCLUSION}

This paper is addressing how a multi-objective control strategy can be developed to obtain multiple independent control variables on a recently introduced self-locking drive concept where simple controllers can act as decentralized controllers. The control variables of interest are the position, the non-load carrying pressure and the drive temperature. Through a decoupling using the load- and level pressures a decoupled system is obtained where it is shown experimentally that a simple proportional controller maintain a maximal position error at 2 $[\mathrm{mm}]$. However, it concluded that the pressure control has spikes when the piston is changing direction. This problem might be due to a too simple pressure controller or disturbances induced by the pressure compensated valves. Regarding the temperature, it is seen that the temperature control works with some steady state error. However, this steady state error may be reduced with another choice of controller. Furthermore, it is concluded that a compromise between the drive stiffness and the temperature is required due to the lack of active cooling. Therefore, if an active cooler in form of a heat exchanger or a fan were mounted on the drive, a more independent temperature control would be achievable since the pressure can be kept high, and thereby the drive stiffness kept high, without compromising the temperature level.

\section{ACKNOWLEDGEMENT}

This research was partially funded by the Danish Energy Agency; the Energy Technology Development and Demonstration Programme (EUDP), project: Energy Efficient and Selfcontained Hydraulic Drive Concept (SenDrive), project number 64018-0094.

\section{NOMENCLATURE}

\begin{tabular}{|c|c|c|}
\hline Variable & Description & Value \\
\hline$A_{A}$ & Piston side area & $31.17\left[\mathrm{~cm}^{2}\right]$ \\
\hline$A_{B}$ & Rod side area & $21.55\left[\mathrm{~cm}^{2}\right]$ \\
\hline$B_{v}$ & Viscous damping & $1053[\mathrm{Ns} / \mathrm{m}]$ \\
\hline$C_{L A}$ & $\begin{array}{l}\text { Pump A leakage } \\
\text { coefficient }\end{array}$ & $\begin{array}{l}3.3 \mathrm{e}-3 \\
{[\mathrm{~L} / \mathrm{min} / \mathrm{bar}]}\end{array}$ \\
\hline$C_{L B}$ & $\begin{array}{l}\text { Pump B leakage } \\
\text { coefficient }\end{array}$ & $\begin{array}{l}1.0 \mathrm{e}-3 \\
{[\mathrm{~L} / \mathrm{min} / \mathrm{bar}]}\end{array}$ \\
\hline$D_{A}$ & Displacement pump A & $16.5 \mathrm{e}-3[\mathrm{~L} / \mathrm{rev}]$ \\
\hline$D_{B}$ & Displacement pump B & $11.3 \mathrm{e}-3$ [L/rev] \\
\hline$L_{x}$ & Piston length & $700[\mathrm{~mm}]$ \\
\hline$M$ & Mass & $700[\mathrm{~kg}]$ \\
\hline$P_{a t m}$ & Atmospherically press. & 1.017 [bar] \\
\hline$V_{A 0}$ & $\begin{array}{l}\text { Dead volume piston } \\
\text { side }\end{array}$ & $0.33[\mathrm{~L}]$ \\
\hline$V_{B 0}$ & Dead volume rod side & $0.12[\mathrm{~L}]$ \\
\hline$V_{A T}$ & AT chamber volume & $0.16[\mathrm{~L}]$ \\
\hline$V_{B T}$ & BT chamber volume & $0.11[\mathrm{~L}]$ \\
\hline$\omega_{e}$ & Motor eigenfrequency & $80 \pi[\mathrm{rad} / \mathrm{s}]$ \\
\hline$\omega_{v}$ & Valve eigenfrequency & $42 \pi[\mathrm{rad} / \mathrm{s}]$ \\
\hline$\zeta_{e}$ & Motor damping & $0.5[-]$ \\
\hline$\zeta_{v}$ & Valve damping & $1[-]$ \\
\hline$K_{\{v A, v B\}}$ & $\begin{array}{l}\text { Flow gain, valve vA } \\
\text { and vB. }\end{array}$ & $8.5\left[\frac{L}{\min \sqrt{b a r}}\right]$ \\
\hline$K_{\{v A T, v B T\}}$ & $\begin{array}{l}\text { Flow gain, valve vAT } \\
\text { and vBT }\end{array}$ & $8.5\left[\frac{L}{\min \sqrt{b a r}}\right]$ \\
\hline$V_{a c c}$ & Accumulator volume & $3.5[\mathrm{~L}]$ \\
\hline$V_{T 0}$ & $\begin{array}{l}\text { Accumulator dead } \\
\text { volume }\end{array}$ & $0.91[\mathrm{~L}]$ \\
\hline$c_{e q}$ & $\begin{array}{l}\text { Equivalent specific } \\
\text { heat capacity for } \\
\text { manifold }\end{array}$ & $1500[\mathrm{~J} / \mathrm{kg} / \mathrm{K}]$ \\
\hline$m_{e q}$ & $\begin{array}{l}\text { Equivalent manifold } \\
\text { mass }\end{array}$ & $19.5[\mathrm{~kg}]$ \\
\hline
\end{tabular}




$\begin{array}{lll}h_{n} & \begin{array}{l}\text { Heat transfer coeff., } \\ \text { natural convection and } \\ \text { radiation part }\end{array} & 7.1[\mathrm{~W} / \mathrm{K}] \\ h_{f} & \begin{array}{l}\text { Heat transfer coeff., } \\ \text { forced convection part }\end{array} & 2.52[\mathrm{~W} / \mathrm{K}] \\ & \end{array}$

\section{REFERENCES}

[1] Ketelsen, S., Padovani, D., Andersen, T., Ebbesen, M., and Schmidt, L. (2019). "Classification and Review of PumpControlled Differential Cylinder Drives". Energies.

[2] Minav, T., Bonato, C., Sainio, P., and Pietola, M. (2014). "Direct Driven Hydraulic Drive". In Proceedings of the $9^{\text {th }}$ International Fluid Power Conference.

[3] Järf, A., Minav, T., and Pietola, M. (2016) "Nonsymmetrical Flow Compensation Using Hydraulic Accumulator". In Proceedings of the 9th FPNI Ph.D. Symposium on Fluid Power.

[4] C. Pedersen, H. C., Schmidt, L., Andersen, T. O., and Brask, M. H. (2014) "Investigation of New Servo Drive Concept Utilizing Two Fixed Displacement Units". JFPS International Journal of Fluid Power System.

[5] Gøytil, P., Padovani, D., and Hansen, M. R. (2019) "On the Energy Efficiency of Dual Prime Mover Pump-Controlled Hydraulic Cylinders". In Proceedings of the ASME/BATH 2019 Symposium on Fluid Power and Motion Control.

[6] Padovani, D., Ketelsen, S., Hagen, D., and Schmidt, L. (2019) "A Self-Contained Electro-Hydraulic Cylinder with Passive Load-Holding Capability", Energies.

[7] Michel, S., and Weber, J. (2012).

"Electrohydraulic Compact-drives for Low Power Applications considering Energyefficiency and High Inertial Loads". In Proceedings of the 7th FPNI PhD Symposium on Fluid Power.

[8] Michel, S., and Weber, J. (2012). "Energyefficient electrohydraulic compact drives for low power applications". In Proceedings of the 2012 Bath/ASME Symposium on Fluid Power and Motion Control.

[9] Caliskan, H., Balkan, T., and Platin, B. E., (2016) "A Complete Analysis for Pump Controlled Single Rod Actuators". In Proceedings 10th International Fluid Power Conference.

[10] Brahmer, B. (2012) "CLDP - Hybrid Drive using Servo Pump in Closed Loop". In
Proceedings of 8th International Fluid Power Conference.

[11] Schmidt, L, Groenkjaer, M, Pedersen, H, Andersen, T (2017) "Position Control of an Over-Actuated Direct Hydraulic Cylinder Drive". Control Engineering Practice.

[12] Schmidt, L., Roemer, D. B., Pedersen, H. C., and Andersen, T. O. (2015) "Speed-Variable Switched Differential Pump System for Direct Operation of Hydraulic Cylinders". In Proceedings of the ASME/BATH 2015 Symposium on Fluid Power and Motion Control.

[13] Ketelsen, S., Schmidt, L., Donkov, V. H., and Andersen, T. O. (2018) "Energy Saving Potential in Knuckle Boom Cranes using a Novel Pump Controlled Cylinder Drive". Modeling, Identification and Control: A Norwegian Research Bulletin.

[14] Schmidt, L., Ketelsen, S., Brask, M. H., and Mortensen, K. A. (2019) "A Class of Energy Efficient Self-Contained Electro-Hydraulic Drives with Self-Locking Capability". Energies.

[15] Schmidt, L, Ketelsen, S., Padovani, D., and Mortensen K. (2019) "Improving the Efficiency and Dynamic Properties of a Flow Unit in a Self-locking Compact ElectroHydraulic Cylinder Drive". In proceedings of ASME/Bath 2019 Symposium on Fluid Power \& Motion Control. 


\section{APPENDIX}
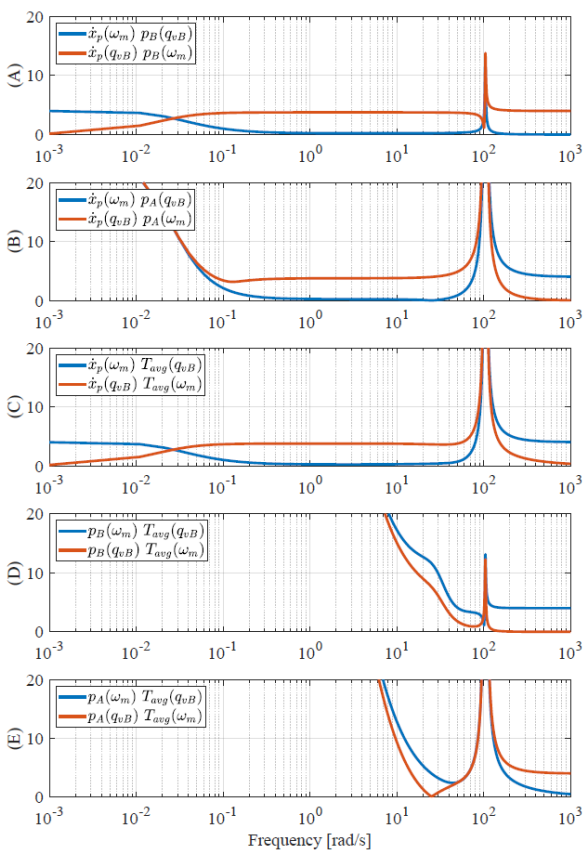

Figure. A1. Relative gain array for system with actuator dynamics and with $x_{p 0}=5[\mathrm{~mm}]$.
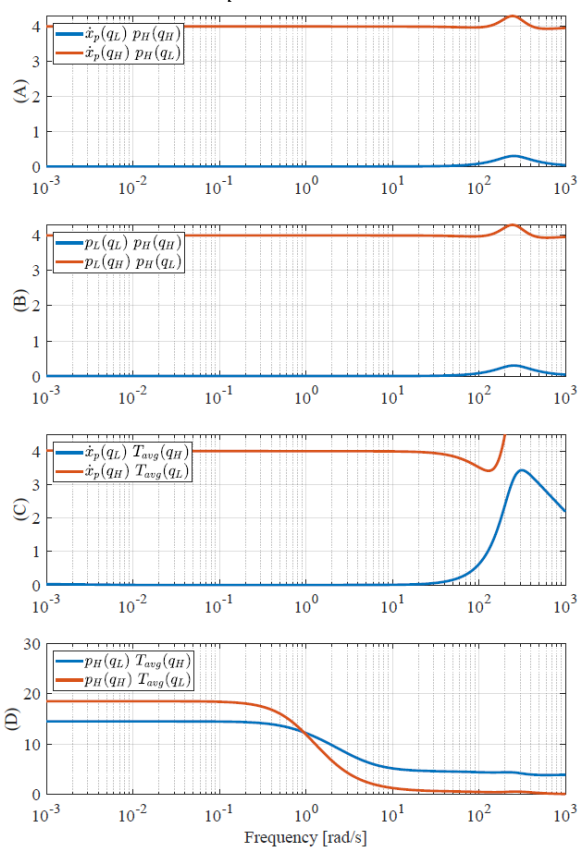

Figure A2. Relative gain array for system with inputand output transformations and with $x_{p 0}=5[\mathrm{~mm}]$.
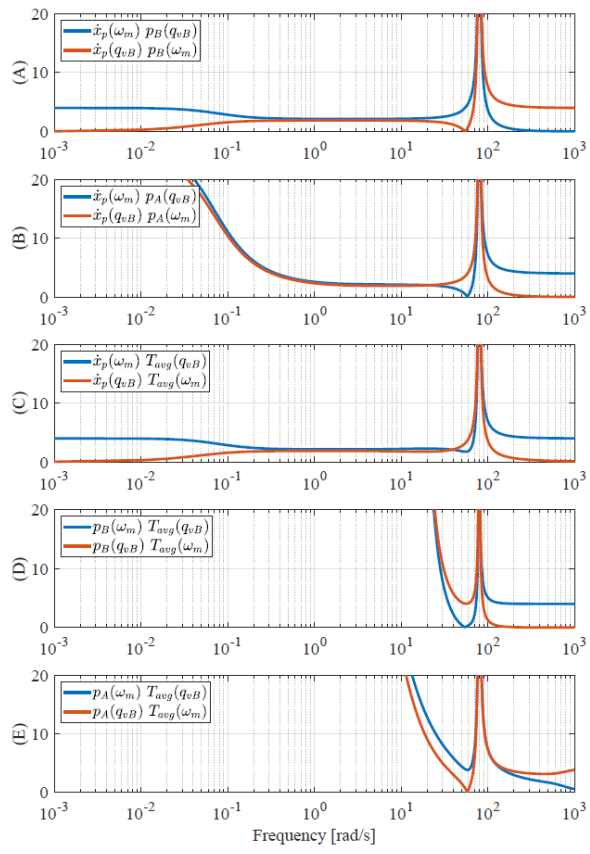

Figure. A3. Relative gain array for system with actuator dynamics and with $x_{p 0}=665[\mathrm{~mm}]$.
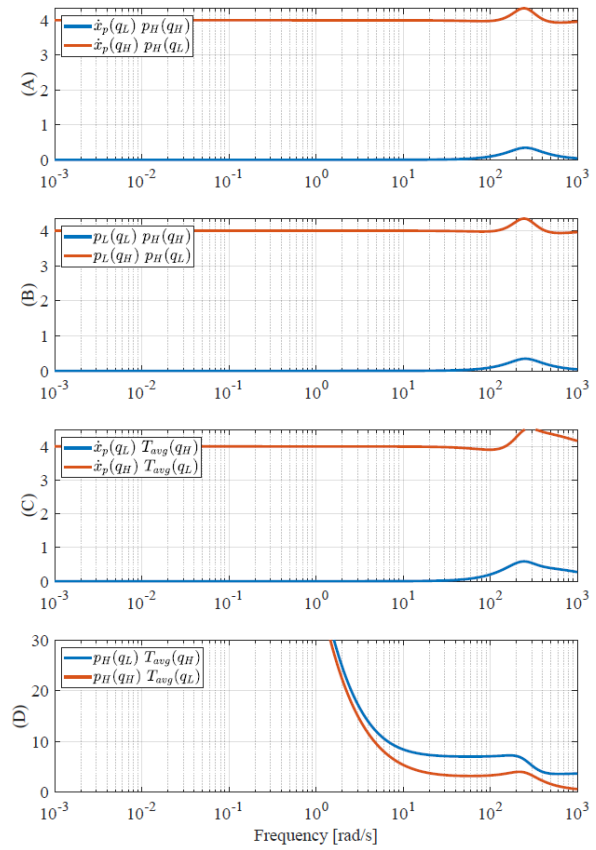

Figure A4. Relative gain array for system with inputand output transformations and with $x_{p 0}=665[\mathrm{~mm}]$. 